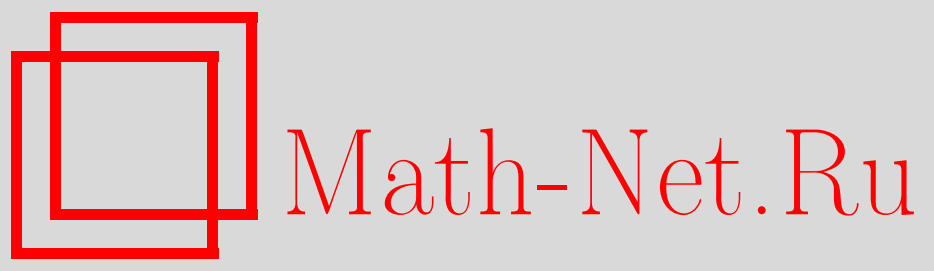

Н. Р. Садыков, Влияние поляризации на траекторию диссипативных солитонов, ТМФ, 2009, том 158, номер 1, 126-134

DOI: https://doi.org/10.4213/tmf6303

Использование Общероссийского математического портала Math-Net.Ru подразумевает, что вы прочитали и согласны с пользовательским соглашением http://www . mathnet.ru/rus/agreement

Параметры загрузки:

IP : 3.95 .254 .165

26 апреля 2023 г., 16:28:35

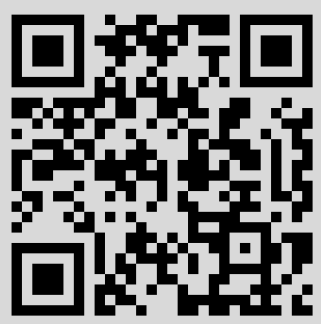




\title{
ВЛИЯНИЕ ПОЛЯРИЗАЦИИ НА ТРАЕКТОРИЮ ДИССИПАТИВНЫХ СОЛИТОНОВ
}

\begin{abstract}
Показано, что для диссипативных солитонов оптический эффект Магнуса определяется не только спиральностью, но и топологическим индексом: магнитным квантовым числом или проекцией орбитального момента солитона на его траекторию. Для неоднородной среды установлена связь между оптическим эффектом Магнуса и неголономностью поля касательных к траектории единичных векторов.
\end{abstract}

Ключевые слова: диссипативные солитоны, оптический эффект Магнуса, влияние поляризации на траекторию.

\section{1. ВВЕДЕНИЕ}

При анализе волновых полей и поляризационных эффектов важную роль играет как метод геометрической оптики, так и волновые методы прикладной электродинамики, которые основаны на методах функций Грина, волноводных мод и др. Геометрическая оптика является простым и наглядным инструментом, обеспечивающим хорошее количественное описание широкого круга волновых явлений различной физической природы в случае, когда длина волны мала по сравнению с характерными масштабами задачи. Геометрическая оптика в узком (лучевом) смысле изучает только способы построения изображений при помощи лучей. В таком понимании построение геометрической оптики было завершено в фундаментальных трудах Гамильтона. В более широком (волновом) понимании геометрическая оптика выступает как метод приближенного описания волновых полей. В этом случае лучи образуют только геометрическую основу, на которую затем "нашивается" волновое поле. Современный волновой период геометрической оптики ведет свое начало с работ Дебая. В рамках такой интерпретации геометрической оптики в последнее время в научных публикациях используют понятие "взаимного влияния" поляризации и траектории [1]. Типичным примером влияния траектории на поляризацию является геометрическая фаза Рытова-Владимирского [2] (прототип фазы

${ }^{*}$ Российский федеральный ядерный центр-Всероссийский научно-исследовательский институт технической физики им. академика Е.И. Забабахина, Снежинск, Челябинская обл., Россия. E-mail: n.r.sadykov@rambler.ru 
Берри). Обратными по отношению к указанному эффекту являются эффекты влияния поляризации на траекторию пучка лучей: оптический эффект Магнуса [3], [4], обратный оптический эффект Магнуса [5] (в приближении геометрической оптики) и др. (см. [1]).

В приближении геометрической оптики оптический эффект Магнуса приводит к дополнительному кручению траектории пучка лучей и определяется кривизной траектории и знаком циркулярной поляризации $\sigma$. Обратный оптический эффект Магнуса приводит к дополнительному изгибу траектории пучка лучей, который пропорционален кручению траектории пучка лучей и также определяется знаком циркулярной поляризации. При выводе уравнения траектории в [1], [4], [5] считалось, что в случае отсутствия нелинейных эффектов (излучение слабой интенсивности) для волны всегда имеет место условие равенства нулю топологического индекса $m$ (в квантовой механике $m$ - магнитное квантовое число). Но современные возможности экспериментальной техники настолько возросли, что сейчас в нелинейных средах в случае мощного лазерного излучения существуют физические объекты, для которых $m \neq 0$ (например, диссипативные солитоны [6]-[11]). Поэтому в общем случае мы можем считать, что в рамках геометрической оптики выполняется условие $m \neq 0$. В случае обратного оптического эффекта Магнуса зависимость от $m$ была получена в работе [5]. Поэтому естественно предположить, что оптический эффект Магнуса также зависит от топологического индекса $m$ (проекции орбитального момента солитона на его траекторию).

В настоящей работе показано, что для диссипативных солитонов оптический эффект Магнуса определяется не только спиральностью $\sigma$, но и топологическим индексом $m$. Для неоднородной среды установлена связь между оптическим эффектом Магнуса и неголономностью поля касательных к траектории единичных векторов.

\section{2. ЗАВИСИМОСТЬ ВЕЛИЧИНЫ ДОПОЛНИТЕЛЬНОГО КРУЧЕНИЯ ТРАЕКТОРИИ ЦИРКУЛЯРНО ПОЛЯРИЗОВАННОГО ПУЧКА ЛУЧЕЙ ОТ ТОПОЛОГИЧЕСКОГО ИНДЕКСА}

В работе [5] в результате анализа векторного волнового уравнения в геликоидальной системе координат (неподвижной относительно трехгранника Френе) было показано, что в случае скрученного многомодового световода с величиной кручения $K=$ const постоянные распространения направляемых мод равны $\beta_{N, m}=$ $\beta_{N, m}^{(0)}-K(\sigma+m)$. После обобщения этих результатов на локально изотропную неоднородную среду из укороченного действия было получено уравнение траектории пучка лучей, описывающее обратный оптический эффект Магнуса, причем величина эффекта определяется полным топологическим зарядом $\sigma+m[5]$. В то же время оптический эффект Магнуса (учет кривизны траектории пучка лучей) определяется только $\sigma$. Покажем, что оптический эффект Магнуса также определяется полным топологическим зарядом $\alpha \sigma+m$, где $\alpha$ - постоянная величина, $\alpha \sim 1$, т.е. поправка к фазе будет зависеть не только от $\sigma$, но и от топологического индекса $m$. При этом следует отметить, что условие $m \neq 0$ означает значительное увеличение оптического объема волноводного канала (см. книгу [12], с. 274). В случае нелинейной среды 
волноводный канал формируется за счет самофокусировки, поэтому требуется мощное лазерное излучение. Только в последние десятилетия появилась возможность наблюдать эффекты, которые определяются топологическим индексом $m$. В случае оптического эффекта Магнуса одним из интересных в этом смысле физических объектов являются диссипативные солитоны [6]-[11].

В оптическом эффекте Магнуса поперечная компонента волнового вектора, обуславливающая этот эффект, определяется параметром траектории - кривизной. Это означает, что волновая функция в каждой точке волнового пакета содержит "добавку", небольшую в масштабе процессов, происходящих в самом пакете, в результате чего траектория центра волнового пакета начинает скручиваться как единое целое. Применительно к диссипативным солитонам это означает, что можно не рассматривать "внутреннюю структуру" солитона. Понятно, что такой малый эффект, как дополнительное кручение траектории пучком лучей, практически не влияет на происходящие внутри солитона процессы, в частности, не влияет на структуру энергетических потоков [11].

Оптические солитоны, представляющие собой сгустки света, линейное (дифракционное и дисперсионное) расплывание которых уравновешено нелинейным сжатием, делятся на два типа. Первый из них соответствует консервативным солитонам, которые формируются и распространяются в прозрачной среде с малыми потерями [6]. Спектр основных характеристик консервативных солитонов сплошной. Второй тип соответствует диссипативным солитонам, или автосолитонам. Диссипативные оптические солитоны были предсказаны и теоретически изучены первоначально в широкоапертурных нелинейных интерферометрах [7] и лазерах с насыщающимся поглощением [8]. По сравнению со случаем консервативных солитонов дополнительное требование энергетического баланса приводит к дискретности спектра основных характеристик диссипативных солитонов. С современным обзором теории и экспериментальных данных для диссипативных солитонов можно ознакомиться в [9]-[11].

В случае двухуровневой модели сред с усилением и поглощением имеет место насыщение поглощения

$$
f\left(|E|^{2}\right)=-1+\frac{g_{0}}{1+|E|^{2}}-\frac{a_{0}}{1+b|E|^{2}},
$$

где функция $f$ определяет нелинейную часть в обобщенном комплексном уравнении Гинзбурга-Ландау [11].

Поскольку имеются нерезонансные слагаемые, можно предположить наличие слабого насыщения дисперсии, т.е. в этом случае диэлектрическую проницаемость мы можем представить в виде

$$
\varepsilon=\varepsilon^{\prime}+i \varepsilon^{\prime \prime}, \quad \varepsilon^{\prime \prime} \ll \varepsilon^{\prime} .
$$

Условие равенства нулю потерь энергии в уравнении баланса означает, что “волновое число" диссипативного солитона является вещественной величиной, именно этим вызвано применение уравнения для комплексной радиальной функции в работе [11] при анализе внутренней структуры такого солитона (см. далее формулу (8)). Воспользуемся аналогичным приближением, но в отличие от [11] анализ, во-первых, 
проведем по аналогии с [4] (см. также [1]) в ортогональной тороидальной (изогнутой) системе координат:

$$
\begin{gathered}
x^{1}=(R+r \cos \varphi) \cos \frac{z}{R}, \quad x^{2}=(R+r \cos \varphi) \sin \frac{z}{R}, \quad x^{3}=-r \sin \varphi, \\
h_{1}=1, \quad h_{2}=r, \quad h_{3}=\frac{R+r \cos \varphi}{R} .
\end{gathered}
$$

где $z$ - натуральный параметр (отсчитываемый вдоль оси симметрии диссипативного солитона), $\varphi$ - угол между кривизной и радиусом $\mathbf{r}$ в локально цилиндрической системе координат, центр которой лежит на оси симметрии солитона. Формулы (3) устанавливают связь между переменными $x^{1}, x^{2}, x^{3}$ декартовой системы координат и переменными $r, \varphi, z$ криволинейной системы координат.

Во-вторых, для рассмотрения кручения траектории солитонов воспользуемся не скалярным, а векторным волновым уравнением

$$
\operatorname{rot} \operatorname{rot} \mathbf{E}=\frac{\omega^{2}}{c^{2}}\left(\varepsilon^{\prime}+i \varepsilon^{\prime \prime}\right) \mathbf{E} .
$$

Если же пользоваться скалярным волновым уравнением, то мы получим только деформацию волновой функции вдоль радиуса кривизны траектории уединенного солитона (см. [12], с. 606), которая при большой кривизне изгиба траектории солитона может привести к эффекту “туннелирования".

В работе [4] было показано, что в случае изогнутого волокна постоянная распространения пробной функции направляемой моды содержит бинормальную компоненту, которая определяется знаком циркулярной поляризации $\sigma$. Для этого использовалась система координат (3). По аналогии с работой [4] покажем, что при распространении излучения по изогнутой траектории волновое число (в неоднородной среде оно выполняет роль постоянной распространения) содержит бинормальную компоненту, которая определяется не только $\sigma$, но и топологическим индексом $m$. В нашем случае в отличие от [4] волноводный канал будет формироваться за счет нелинейных эффектов.

Для решения векторного волнового уравнения (4) подберем пробную функцию, воспользовавшись результатами работы [11]. Пробная векторная волновая функция должна быть пропорциональна функции $\left(\mathbf{e}_{x}+i \sigma \mathbf{e}_{y}\right) e^{i(\beta z-\omega t)}$ и содержать для описания диссипативного солитона радиальную функцию, определяемую "радиальным" числом $N$ и "магнитным" числом $m$ (топологическим индексом); здесь $\mathbf{e}_{x}, \mathbf{e}_{y}-$ единичные векторы локально декартовой системы координат. При изгибе, кроме параметра, характеризующего деформацию волновой функции вдоль радиуса кривизны траектории, волновая функция должна содержать параметр, характеризующий наличие поперечной составляющей у волнового числа [4], [13]. С учетом всего вышесказанного зададим пробную функцию как

$$
\begin{aligned}
\mathbf{E}(r, \varphi, z) & =\mathbf{E}_{0} e^{i(\beta z-\omega t)}=\frac{\mathbf{e}_{x}+i \sigma \mathbf{e}_{y}}{\sqrt{2}} \Psi_{m N}(r, \varphi) e^{i(\beta z-\omega t)}, \\
\Psi_{m N}(r, \varphi) & =F_{m N}(r) e^{i m \varphi+r(\kappa \cos \varphi+i \eta \sin \varphi)},
\end{aligned}
$$

5 Теоретическая и математическая физика, т. 158, № 1, 2009 г. 
где параметры $\kappa$ и $\eta$ характеризуют соответственно деформацию волновой функции вдоль радиуса кривизны траектории и наличие поперечной составляющей волнового числа. Выразим векторы $\mathbf{e}_{x}, \mathbf{e}_{y}$ через единичные векторы локально цилиндрической системы координат $\mathbf{e}_{r}, \mathbf{e}_{\varphi}$ следующим образом:

$$
\begin{gathered}
\mathbf{e}_{x}=\mathbf{e}_{r} \cos \varphi-\mathbf{e}_{\varphi} \sin \varphi, \quad \mathbf{e}_{y}=\mathbf{e}_{r} \sin \varphi+\mathbf{e}_{\varphi} \cos \varphi \\
r^{2}=x^{2}+y^{2}, \quad x=r \cos \varphi, \quad y=r \sin \varphi
\end{gathered}
$$

Отсюда следует, что

$$
\mathbf{e}_{x}+i \sigma \mathbf{e}_{y}=\left(\mathbf{e}_{r}+i \sigma \mathbf{e}_{\varphi}\right) e^{i \sigma \varphi} .
$$

В представлении (5) скалярная волновая функция $F_{m N}(r)$ удовлетворяет нелинейному уравнению [11]:

$$
\frac{\partial^{2} F_{m N}}{\partial r^{2}}+\frac{1}{r} \frac{\partial}{\partial r} F_{m N}-\frac{m^{2}}{r^{2}} F_{m N}+\left[i \nu+f\left(\left|F_{m N}\right|^{2}\right)\right] \frac{F_{m N}}{i+d}=0,
$$

где $\left.F_{m N}(r)\right|_{r=0}=0,\left.F_{m N}(r)\right|_{r \rightarrow \infty}=0, d-$ эффективный коэффициент диффузии, описывающий слабую дисперсию среды, $0<d \ll 1, \nu$ - частотный сдвиг для стационарного солитона, являющийся собственным значением задачи (масштаб $\nu$ равен обратному значению времени затухания в пустом резонаторе), функция $f$ определена в (1).

По аналогии с работой [4] с учетом (3), (5) и (7) получаем

$$
\operatorname{rot} \mathbf{E}=\left[\frac{\sigma \beta}{1+r \cos \varphi / R} \mathbf{E}_{0}+\frac{i \sigma \mathbf{e}_{z}}{\sqrt{2}}\left(\frac{\partial}{\partial r}+\frac{1}{r}+i \sigma \frac{\partial}{r \partial \varphi}\right) \Psi_{m N}\right] e^{i(\beta z-\omega t)} .
$$

При $x / r \ll 1, y / r \ll 1$ с учетом (3) из формулы (9) получаем

$$
\begin{aligned}
\operatorname{rot} \operatorname{rot} \mathbf{E}=[ & \frac{\beta^{2}}{(1+r \cos \varphi / R)^{2}} \mathbf{E}_{0}-\frac{\mathbf{e}_{x}+i \sigma \mathbf{e}_{y}}{\sqrt{2}} \Delta_{\perp}^{\prime} \Psi_{m N}+\vec{\nabla}_{\perp}^{\prime}\left(\vec{\nabla}_{\perp}^{\prime} \mathbf{E}_{0}\right)- \\
& -i \frac{\sigma}{R} \frac{\mathbf{e}_{y}}{\sqrt{2}}\left(\frac{\partial \Psi_{m N}}{\partial x}+i \sigma \frac{\partial \Psi_{m N}}{\partial y}\right)+ \\
& \left.+\frac{i \beta \mathbf{e}_{z} / \sqrt{2}}{1+r \cos \varphi / R}\left(\frac{\partial \Psi_{m N}}{\partial x}+i \sigma \frac{\partial \Psi_{m N}}{\partial y}\right)\right] e^{i(\beta z-\omega t)},
\end{aligned}
$$

где

$$
\Delta_{\perp}^{\prime}=\frac{\partial^{2}}{\partial x^{2}}+\frac{\partial^{2}}{\partial y^{2}}, \quad \vec{\nabla}_{\perp}^{\prime}=\mathbf{e}_{x} \frac{\partial}{\partial x}+\mathbf{e}_{y} \frac{\partial}{\partial y}
$$

и учтено, что

$$
\begin{gathered}
\frac{i \sigma}{\sqrt{2}}\left(\mathbf{e}_{r} \frac{\partial}{r \partial \varphi}-\mathbf{e}_{\varphi} \frac{\partial}{\partial r}\right)\left[\frac{\partial \Psi_{m N}}{\partial r}+\frac{\Psi_{m N}}{r}+i \sigma \frac{\partial \Psi_{m N}}{r \partial \varphi}\right]= \\
=-\frac{\mathbf{e}_{x}+i \sigma \mathbf{e}_{y}}{\sqrt{2}} \Delta_{\perp}^{\prime} \Psi_{m N}+\vec{\nabla}_{\perp}^{\prime}\left(\vec{\nabla}_{\perp}^{\prime} \mathbf{E}_{0}\right)
\end{gathered}
$$

при выводе равенства (10) учтены также соотношения (6). 
С учетом (10) векторное волновое уравнение (4) может быть записано в виде

$$
\begin{aligned}
\frac{\mathbf{e}_{x}+i \sigma \mathbf{e}_{y}}{\sqrt{2} \Delta_{\perp}^{\prime} \Psi_{m N}} & -\frac{\beta^{2}}{(1+x / R)^{2}} \mathbf{E}_{0}-\vec{\nabla}_{\perp}^{\prime}\left(\vec{\nabla}_{\perp}^{\prime} \mathbf{E}_{0}\right)+ \\
& +i \frac{\sigma}{R} \frac{\mathbf{e}_{y}}{\sqrt{2}}\left(\frac{\partial \Psi_{m N}}{\partial x}+i \sigma \frac{\partial \Psi_{m N}}{\partial y}\right)- \\
& -\frac{i \beta \mathbf{e}_{z} / \sqrt{2}}{1+x / R}\left(\frac{\partial \Psi_{m N}}{\partial x}+i \sigma \frac{\partial \Psi_{m N}}{\partial y}\right)+\frac{\omega^{2}}{c^{2}}\left(\varepsilon^{\prime}+i \varepsilon^{\prime \prime}\right) \mathbf{E}_{0}=0
\end{aligned}
$$

Умножим это уравнение на $\left(\mathbf{e}_{x}-i \sigma \mathbf{e}_{y}\right) / \sqrt{2}$. При $r \ll R$ получаем

$$
\begin{aligned}
& {\left[\frac{\partial^{2} F_{m N}}{\partial r^{2}}+\frac{1}{r} \frac{\partial}{\partial r} F_{m N}-\frac{m^{2}}{r^{2}} F_{m N}+\frac{\omega^{2}}{c^{2}}\left(\varepsilon^{\prime}+i \varepsilon^{\prime \prime}\right) F_{m N}-\beta^{2} F_{m N}+\right.} \\
& \left.\quad+\frac{\mathbf{e}_{x}-i \sigma \mathbf{e}_{y}}{\sqrt{2}} \vec{\nabla}_{\perp}^{\prime}\left(\vec{\nabla}_{\perp}^{\prime} \mathbf{E}_{0}\right)\right]+i\left\{\frac{\sigma}{2 R} \frac{\partial F_{m N}}{\partial y}+2 \eta \frac{\partial F_{m N}}{\partial y}-\frac{m}{2 R} \frac{y}{r^{2}} F_{m N}\right\}+ \\
& \quad+\left\{2 \kappa \frac{\partial F_{m N}}{\partial x}+\frac{1}{2 R} \frac{\partial F_{m N}}{\partial x}+2 \frac{\beta^{2} x}{R} F_{m N}-\frac{\sigma m}{2 R} \frac{x}{r^{2}} F_{m N}\right\}=0
\end{aligned}
$$

где учтено, что (см. также (6))

$$
\frac{\partial}{\partial x}+i \sigma \frac{\partial}{\partial y}=e^{i \sigma \varphi}\left[\frac{\partial}{\partial r}+i \sigma \frac{\partial}{r \partial \varphi}\right]
$$

Выражение в квадратных скобках в (14) эквивалентно левой части (8), т.е. равно нулю. При этом в данном выражении в отличие от (8) имеется поляризационное слагаемое (последнее слагаемое), учет которого в соответствии с хорошо разработанной теорией волноводных мод приведет к незначительной корректировке величины $\beta^{2}$ в (14) (см. [12], с. 260). Кроме того, при выводе (14) мы пренебрегли слабой пространственной дисперсией (в $(8)$ положили $d=0)$. Выражение во вторых фигурных скобках в (14) приводит к деформации волновой функции вдоль радиуса кривизны траектории [1], [4], а выражение в первых фигурных скобках в (14) - к наличию поперечной составляющей волнового числа диссипативного солитона. Для оценки величины поперечной составляющей поступим следующим образом. Пусть выражение в первых фигурных скобках после умножения на $F_{m N}^{*} \sin \varphi$ $\left(F_{m N}^{*}-\right.$ комплексно-сопряженная величина к $\left.F_{m N}\right)$ и интегрирования по поперечному сечению равняется нулю. Тогда получаем

$$
i \int r \sin ^{2} \varphi\left[\left(\frac{\sigma}{2 R}+2 \eta\right) F_{m N}^{*} \frac{\partial F_{m N}}{\partial r}-\frac{m}{2 R} \frac{\left|F_{m N}\right|^{2}}{r}\right] d r d \varphi=0 .
$$

С учетом краевых условий для $F_{m N}($ см. (8)) это равенство эквивалентно равенству

$$
\int\left(\frac{\sigma}{2 R}+2 \eta+\frac{m}{R}\right)\left|F_{m N}\right|^{2} d r=0 .
$$

Отсюда получаем приближенное значение величины $\eta$, которая характеризует наличие поперечной составляющей у волнового числа диссипативного солитона:

$$
\eta=-\frac{\sigma}{4 R}-\frac{m}{2 R}
$$


Далее, поступая аналогично работам [1], [4], из (18) получаем укороченное действие для диссипативного солитона (принцип Ферма)

$$
\int_{1}^{2}\left(\beta d s+A_{\alpha} d x^{\alpha}\right)
$$

где $\beta=n k, k=2 \pi / \lambda, \lambda$ - длина волны излучения в вакууме, $n$ - показатель преломления; $s$ - натуральный параметр, отсчитываемый вдоль траектории солитона; $A_{\alpha}-\alpha$-я компонента вектора $\mathbf{A}=-(\sigma / 4+m / 2) \mathbf{l} \times \boldsymbol{\tau}, \boldsymbol{\tau}=\mathbf{R} / R^{2}=-\mathbf{l} \times \vec{\nabla} \ln n \times \mathbf{l}$; $R=|\mathbf{R}| ; \mathbf{R}$ - радиус кривизны траектории в рассматриваемой точке; $\mathbf{l}$ - касательный к траектории единичный вектор.

Приравняв вариацию от действия (19) нулю, получаем уравнение траектории для диссипативного солитона в неоднородной локально изотропной среде, аналогичное уравнению [1]:

$$
\frac{d \mathbf{l}}{d s}=\mathbf{l} \times \vec{\nabla} \ln n \times \mathbf{l}+\frac{1}{k n} \mathbf{l} \times \operatorname{rot} \mathbf{A} .
$$

С учетом сказанного во введении и аналогично работе [5] уравнение траектории диссипативного солитона (20) при наличии кручения $K$ запишем в виде

$$
\frac{d \mathbf{l}}{d s}=\frac{\mathbf{l} \times \vec{\nabla} \ln n \times \mathbf{l}}{1-K(\sigma+m)}+\frac{1}{k n} \mathbf{l} \times \operatorname{rot} \mathbf{A} .
$$

Это уравнение совпадает с уравнением траектории пучка лучей, полученным в [1], [4] для более простых случаев (при $K=0, m=0$ ) и описывающим в приближении геометрической оптики оптический эффект Магнуса. Кроме того, уравнение (21) совпадает с уравнением траектории пучка лучей спиновых частиц [13], описывающим оптический эффект Магнуса для таких частиц (в последнем случае $\mathbf{A}=-\mathbf{n} \times \boldsymbol{\tau} / 2$, где $\mathbf{n}$ - единичный вектор, направленный вдоль поляризации спиновой частицы).

Уравнение (21) при $\mathbf{A}=0, m=0, K=0$ совпадает с полученным в [14] уравнением траектории пучка лучей для спиновых частиц (и описывает обратный оптический эффект Магнуса для спиновых частиц), если в правой части (21) в знаменателе первого слагаемого вместо величины $\sigma+m$ взять величину $\mathbf{l} \cdot \mathbf{n} / 2$, т.е. величину проекции спина на траекторию частицы. Естественным обобщением результата работы [14] была бы замена величины $\sigma+m$ на величину $\mathbf{l} \cdot \mathbf{J}$, где $\mathbf{J}-$ полный момент спиновой частицы.

\section{3. СВЯЗЬ ОПТИЧЕСКОГО ЭФФЕКТА МАГНУСА С НЕГОЛОНОМНОСТЬЮ ПОЛЯ КАСАТЕЛЬНЫХ К ТРАЕКТОРИИ ЕДИНИЧНЫХ ВЕКТОРОВ}

В соответствии с [15], [16] векторное поле касательных к траектории единичных векторов образует голономное векторное поле [17], т.е. определяет систему кривых (нормальную конгруэнцию, см. книгу [16], с. 154). Это приводит к тому, что можно провести к пучку лучей семейство ортогональных поверхностей, пересекающих каждую кривую под прямым углом. Такие ортогональные поверхности отождествляются с волновыми фронтами (см. [16], с. 812) или волновыми поверхностями 
(см. [16], с. 41). Выполнение условия голономности для поля касательных к траектории единичных векторов приводит к тому, что в оптике имеет место инвариант Лагранжа (см. [16], с. 155).

Из (18) и (19) видно, что в случае оптического эффекта Магнуса возникает аналогия с движением заряженной частицы в электромагнитном поле, где вектор $\mathbf{A}$ играет роль векторного потенциала, а величина $\sigma+m / 2-$ роль заряда. Известно, что при движении заряженной частицы в магнитном поле имеется существенное различие по сравнению с частным случаем чистого электрического поля (см. [16], с. 811). В этом случае существует обобщение инварианта Лагранжа (см. [16], с. 805) одного из инвариантов Пуанкаре. Наличие магнитного поля приводит к тому, что обобщенный импульс содержит векторный потенциал, ротор от которого равен вектору магнитной индукции (в (19) это вектор А). В свою очередь это приводит к неголономности касательного к траектории векторного поля (к косой конгруэнции (см. [16], с. 154)). Действительно, мерой неголономности касательного к траектории единичного векторного поля будет величина l- $\operatorname{rot} \mathbf{l}$ (см. монографию [17], с. 12). Из (20) видно, что при $\mathbf{A}=0$ величина неголономности будет равна нулю, $\mathbf{l} \cdot \operatorname{rot} \mathbf{l}=0$, а при $\mathbf{A} \neq 0$ величина $\mathbf{l} \cdot \operatorname{rot} \mathbf{l} \neq 0$.

\section{4. ОБСУЖДЕНИЕ РЕЗУЛЬТАТОВ}

Итак, эффект дополнительного кручения траектории луча при наличии переменной кривизны траектории (в приближении геометрической оптики - оптический эффект Магнуса) зависит как от знака циркулярной поляризации, так и от топологического индекса $\alpha \sigma+m$, где $\alpha \sim 1$. В этом случае существует качественная аналогия с эффектом дополнительного изгиба при наличии кручения $\sigma+m$ (в приближении геометрической оптики это обратный оптический эффект Магнуса). Количественное отличие, по-видимому, объясняется следующим моментом. В настоящей работе для вывода поперечной составляющей волнового числа (18) использовался подход, аналогичный подходу в [4]. В то же время в работе [18] (или работе [1]) показано, что в действительности величина $\eta$ при $m=0$ будет в два раза больше. Поэтому в (18) скорее всего реализуется соотношение $\eta \sim \sigma+m$. Этот анализ выходит за рамки настоящей работы. Результаты, полученные в работе, применимы к слабовзаимодействующим диссипативным солитонам, когда взаимодействие солитонов между собой аналогично наличию "неоднородной среды" для каждого из взаимодействующих солитонов.

\section{Список литературы}

[1] Н. Р. Садыков, ТМФ, 149:1 (2006), 65-79.

[2] В. В. Владимирский, ДАН СССР, 31 (1941), 222; С. М. Рытов, ДАН СССР, 18 (1938), 263-266.

[3] А. В. Дугин, Б. Я. Зельдович, Н. Д. Кундикова, В. С. Либерман, ЖЭТФ, 100:5(11) (1991), 1474-1482.

[4] Н. Р. Садыков, Квантовая электроника, 19:10 (1992), 1021-1023.

[5] Н. Р. Садыков, Onm. и спектр., 84:4 (1998), 589-597. 
[6] В. Е. Захаров, С.В. Манаков, С. П. Новиков, Л. П. Питаевский, Теория солитонов: Метод обратной задачи, Наука, М., 1980.

[7] Н. Н. Розанов, Г. В. Ходова, Опm. и спектр., 65:6 (1988), 1375-1377.

[8] Н. Н. Розанов, С. В. Федоров, Onm. и спектр., 72:6 (1992), 1403-1408.

[9] V. B. Taranenko, C. O. Weiss, IEEE J. Sel. Top. Quantum Electron, 8:3 (2002), 488-496.

[10] N. N. Rosanov, Spatial Hysteresis and Optical Patterns, Springer, Berlin, 2002.

[11] Н. Н. Розанов, С. В. Федоров, А. Н. Шацев, ЖЭТФ, 125:3 (2004), 486-498.

[12] А. Снайдер, Дж. Лав, Теория оптических волноводов, Радио и связь, М., 1987.

[13] Н. Р. Садыков, ТМФ, 135:2 (2003), 280-288.

[14] Н. Р. Садыков, ТМФ, 144:3 (2005), 555-563.

[15] Ю. А. Кравцов, Ю. И. Орлов, Геометрическая оптика неоднородных сред, Наука, М., 1980.

[16] М. Борн, Э. Вольф, Основы оптики, Наука, М., 1970.

[17] Ю. А. Аминов, Геометрия векторного поля, Наука, М., 1990.

[18] Н. Р. Садыков, Квантовая электроника, 20 (1993), 1140-1142.

Поступила в редакцию 4.09.2007, после доработки 9.03.2008 